\title{
The effect of a postoperative quality improvement program on outcomes in colorectal surgery in a community hospital
}

\author{
C. C. M. Marres ${ }^{1}$ - A. W. H. van de Ven ${ }^{1,2}$ - P. C. M. Verbeek ${ }^{1}$ - S. van Dieren ${ }^{2}$ \\ W. A. Bemelman ${ }^{2}$. C. J. Buskens ${ }^{2}$
}

Accepted: 20 June 2016 / Published online: 6 July 2016

(C) The Author(s) 2016. This article is published with open access at Springerlink.com

\begin{abstract}
Purpose The aim of this study was to evaluate whether implementation of a comprehensive quality improvement program was associated with improved outcomes in patients undergoing oncological colorectal surgery in a non-academic, non-referral community hospital.

Methods The quality improvement program (QIP) was introduced in January 2011 and consisted of the following interventions: (1) avoidance of postoperative nonsteriodal antiinflammatory drugs; (2) normovolemia was pursued preand postoperatively; (3) non-resectional surgery if possible, in patients over 80 with ASA 3 or 4 classification; and (4) a standardized, postoperative surveillance protocol was introduced, with CRP determination day 2 and 4, and if necessary subsequent abdominal $\mathrm{CT}$ with rectal contrast to reduce delay in diagnosis of complications. From a prospectively maintained database of 488 patients undergoing colorectal surgery between 2009 and 2014, postoperative outcomes of patients operated before and after implementation of the program were compared.

Results The severe complication rate (Clavien-Dindo $>3 \mathrm{~b}$ ) decreased significantly $(25.0$ vs. $13.7 \% ; p<.001)$ after implementation of the QIP program. The mortality rate dropped from 8.7 to $2.6 \%(p=.003)$. The percentage of anastomotic
\end{abstract}

C. C. M. Marres

cmarres@flevoziekenhuis.nl

1 Department of Surgery, Flevoziekenhuis, Hospitaalweg 1, 1315, RA Almere, the Netherlands

2 Department of Surgery, Academisch Medisch Centrum, Amsterdam, the Netherlands leakage was 9.6\% before QIP implementation and $4.2 \%$ after $(p=.013)$. Median length of hospital stay decreased from 9 (IQR 5-19) to 7 days (IQR 4-12) $(p<.001)$. Multivariate analyses showed that surgery after implementation of the program was a strong independent predictor for less major complications (OR 0.54, $95 \%$ CI 0.32-0.88).

Conclusions A significant decrease in major complications and mortality was observed after introduction of a relative simple quality improvement program.

Keywords Colorectal surgery $\cdot$ Colorectal resection . Oncology $\cdot$ Quality improvement · Complications

\section{Introduction}

Oncological colorectal surgery carries a moderately high risk for morbidity and mortality. In the literature, the incidence of major complications range from 20 to $35 \%$ and the 30 -day mortality rate from 2 to $9 \%[1-3]$.

During a national clinical audit on oncological colorectal surgery in 2010 in The Netherlands, a relatively high complication and mortality rate was seen in our hospital compared to overall national outcomes [4]. Therefore, after careful re-evaluation of the auditing results and an extensive literature analyses for factors influencing colorectal complications, a comprehensive quality improvement program was composed and introduced in January 2011 to decrease complications and mortality. The aim of this study was to evaluate whether implementation of this program was associated with improved outcomes in patients undergoing oncological colorectal surgery in a non-academic, non-referral community hospital. 


\section{Methods}

\section{Study design}

A cohort study was designed from a prospectively maintained database of patients undergoing an oncological colorectal resection between 2009 and 2014. Patients operated before and after implementation of the QIP program were compared. The institutional review board approved this study.

\section{Establishment of the quality improvement program (QIP)}

A systematic literature search was performed at the end of 2010 using PubMed and Embase. Factors possibly influencing colorectal surgery outcomes were identified and were summarized in Table 1. The literature for all these different factors was compared to our treatment protocol at the time. Each factor was analyzed to see if there was room for improvement in our protocol. Some factors were simply not feasible or applicable in our hospital, such as routinely intraoperative leak testing. In other factors, our treatment protocol

Table 1 Factors that might have impact on postoperative complications

Factors that cannot be changed

Age

Gender

ASA classification

Urgency operation

Type of resection

Stage of cancer

Obesity

Changeable factors

Preoperative mechanical bowel preparation

Experience of surgeon/specialized

Laparoscopic or open procedure

Technique anastomose

Intra-operative leak testing

Protective stoma

Pelvic drain

Operating time, operative strategy

Prophylactic antibiotics

Thrombose prophylactic

Postoperative analgesia (NSAID, opioids)

Fast track recovery protocols

Biomarkers for early diagnosis of complication was in concordance with the optimal treatment according to the literature. Eventually, four factors remained, which seemed suitable for improvement and the following interventions were implemented in our postoperative protocol: Firstly, the use of postoperative nonsteriodal anti-inflammatory drugs was stopped [5-7]. Secondly, normovolemia was pursued preand post-operatively [8-10]. Furthermore, acute resections (with immediate anastomosis) in patients over 80 years with ASA 3 or 4 classification were avoided [11]. In case a frail, high risk patient presented with acute, colonic obstruction, the aim was to manage the obstruction with a colostomy in the acute phase and only perform resection of the tumor in elective setting after optimization of the patient [12-14]. Finally, a standardized postoperative surveillance protocol to reduce delay in diagnosis of complications was introduced; standardized serum C-reactive protein (CRP) level determination on day 2 and 4, if day 4 CRP showed an increase of 50 or an elevation above $200 \mathrm{mg} / \mathrm{L}$, an abdominal CT with rectal contrast was performed $[15,16]$.

With respect to other factors possibly influencing outcomes, the perioperative approach remained unchanged during the timeframe of the study. Postoperatively, patients were managed according to ERAS fast track protocol [17]. Preparation for surgery was the same for all patients. No bowel preparation was given, except for patients undergoing left colonic and rectal resections $[18,19]$. All colorectal resections were performed by or under supervision of specialized colorectal surgeons [20]. Anastomoses were made with a stapler device. In patients who underwent a hemicolectomy, a side-toside anastomose was established and a side-to-end in patients who underwent sigmoid and rectal resections, through the low tie technique (ligation at the level of the superior rectal artery, just caudally to the origin of the left colic artery) [21]. Intraoperative leak testing was only done in very high suspicious situations in low rectal anastomoses through an air leak test. Defunctioning stomas and drain tubes were provided subjective to the surgeon's opinion of the quality of the anastomoses during surgery. Antibiotic prophylaxis was given trough cefazolin $1000 \mathrm{mg}$ plus metronidazole $500 \mathrm{mg}$ intravenously 30 min prior to surgery. If the operation took longer than $3 \mathrm{~h}$, this was repeated. All patients received low-molecular-weight heparin daily during admission to prevent venous thromboembolism [22].

\section{Study population and data collection}

A cohort study was designed from a prospectively maintained database of patients undergoing an oncological colorectal resection in our institution between 2009 and 2014. Patients who underwent transanal endoscopic microsurgery were excluded. The medical records of 484 patients were reviewed. The prospectively maintained database with patient characteristics was supplemented with information on CRP levels, 
timing, and outcome for postoperative CT scans of the abdomen and following interventions. Patients were divided into two cohorts for comparison: those operated before and after implementation of the QIP program in January 2011.

\section{Outcome measures}

The primary outcome measure of interest was major complication. Complications were graded according to the ClavienDindo classification [23], and severe complications being defined as grade $3 \mathrm{~b}$ or higher. Secondary outcome parameters were all postoperative complications, 30-day mortality, failure-to-rescue [24], length of hospital stay, and timing of $\mathrm{CT}$ scans and of re-interventions. Failure-to-rescue rates were defined as mortality among patients with severe complications.

\section{Statistical analysis}

Statistical analysis was performed using IBM SPSS Statisitics for Windows, version 22.0 (IBM corp., Chicago, IL). Continuous variables were presented as mean values with a standard deviation (SD) or as median values with an interquartile range (IQR) according to the distribution (KolmogorovSmirnov and Sapiro-Wilk test). Discrete variables were presented as counts and percentages. Categorical data were compared between groups using the $\chi^{2}$ test, and continuous data were compared using the independent samples $t$ test or MannWhitney $U$ test. Univariate and stepwise multivariate logistic regression models were used to examine the association between the QIP program implementation and severe postoperative complications while adjusting for important potentially confounding variables. The limited number of events (from a statistical point of view) meant that only a restricted number of possible confounders could be examined. Therefore, variables with multiple categories were recorded into dichotomous variables by combining categories with a comparable prognosis (ASA I/II vs. III/IV, radicality R0 vs. R1 and R2, and Dukes A-B vs. C-D).

Clinical important variables based on literature were included in the univariate and multivariate models, as well as all variables with a univariate $p$ value $<.20$. A two-tailed $p$ value of $<.05$ was considered statistically significant.

\section{Results}

\section{Patient demographics}

There were $265(54.8 \%)$ men and 219 (42.2\%) women analyzed in this study with a mean age of 66 years (range 28-89). Relevant patient demographics were summarized in Table 2. Univariate analyses demonstrated no significant differences between the two cohorts concerning age, BMI, sex, ASA classification, tumor stage, stoma or no stoma, and urgency of the operation. There were significantly more minimally invasive procedures in the cohort operated after the QIP program implementation, as expected due to the longitudinal setting of this study. Also, a significant difference in type of resection between the two cohorts was found, with more radical resections in the later patient group. These differences were taken into account in the multivariate analyses. Missing data were for every variable less than $5 \%$, and therefore, there was no imputation of missing data.

\section{Outcome}

The severe complication rate (Clavien-Dindo $>3 \mathrm{~b}$ ) in the first cohort was $25.0 \%$, which significantly decreased to $13.7 \%$ ( $p=.001)$ after implementation of the quality program. The mortality rate decreased from 8.7 to $2.6 \%(p=.003)$. The percentage of anastomotic leakage was $9.8 \%$ before and $4.2 \%$ after $2011(p=.013)$. Median length of hospital stay before implementation of the program was median 9 (IQR 5$19)$ and after implementation 7 (IQR 4-12) $(p<.001)$. The minor complication rate (Clavien-Dindo $<3 b$ ) was not significantly different in the two cohorts (respectively 24.4 and $25.3 \%$ ) (Table 3 ).

\section{Multivariate analysis}

In the multivariate analysis (Table 4), all clinically and statistically relevant $(p<.20$ in univariate models for the outcome) factors were included: elective versus emergency resection, age at time of operation, ASA classification, stoma vs. no stoma, type of resection, open or minimally invasive procedures, and the date of the operation (before or after implementation of the program). Multivariate analyses showed that beside these factors, the date of the operation was a strong independent predictor for a major complication (OR $0.53,95 \% \mathrm{CI}$ 0.32-0.88).

\section{High risk elderly patients}

Before 2011,18 patients ( $10.5 \%$ of total) undergoing surgery were aged 80 years or over with an ASA 3 or 4 classification. After 2011, 20 patients (6.4\%) with these characteristics were operated upon. A primary anastomosis was made in 14 out of 18 patients $(78 \%)$, and after 2011,11 out of $20(55 \%)$ received a primary anastomosis.

\section{CT abdomen and early intervention}

The interval between operation and first CT was 9.0 days before 2011 and 6.0 days after 2011 ( $p=.553$; median 7 vs. 6 days). The time until re-intervention decreased from 9.6 days 
Table 2 Patient demographics

\begin{tabular}{|c|c|c|c|c|}
\hline Variables & $\begin{array}{l}\text { Pre-implementation } \\
N(\%)^{\mathrm{a}}\end{array}$ & $\begin{array}{l}\text { Post-implementation } \\
N(\%)^{\mathrm{a}}\end{array}$ & $\begin{array}{l}\text { Total } \\
N(\%)^{\mathrm{a}}\end{array}$ & $p$ value \\
\hline Age & & & & $.283^{\mathrm{b}}$ \\
\hline Average (SD) & $67(13)$ & $66(11)$ & $66(12)$ & \\
\hline Range & $30-96$ & $30-90$ & $28-89$ & \\
\hline Sex & & & & $.545^{\mathrm{c}}$ \\
\hline Female & $81(47.1)$ & $138(44.2)$ & $219(45.2)$ & \\
\hline Male & $91(25.9)$ & $174(55.8)$ & $265(54.8)$ & \\
\hline BMI & & & & $.989^{\mathrm{b}}$ \\
\hline Average (SD) & $26.3(4.2)$ & $26.3(4.5)$ & $26.3(4.4)$ & \\
\hline Range & $16.5-45.3$ & $16.2-50.2$ & $16.2-50.2$ & \\
\hline ASA & & & & $.246^{\mathrm{c}}$ \\
\hline ASA 1 or 2 & $130(75.6)$ & $251(80.4)$ & 381 (78.7) & \\
\hline ASA 3 or 4 & $42(24.4)$ & $61(19.6)$ & $103(21.3)$ & \\
\hline Type of operation & & & & $.006^{\mathrm{c}}$ \\
\hline Right hemicolectomy & $49(28.5)$ & 109 (34.9) & $158(32.6)$ & \\
\hline Left hemicolectomy & $12(7.0)$ & $24(7.7)$ & $36(7.4)$ & \\
\hline Sigmoidectomy/LAR & $87(50.6)$ & 139 (44.6) & $226(46.7)$ & \\
\hline Colectomy & $2(1.2)$ & $18(5.8)$ & $20(4.1)$ & \\
\hline APR & $12(7.0)$ & $19(6.1)$ & $31(6.4)$ & \\
\hline Other & $10(5.8)$ & $3(1.0)$ & $13(2.7)$ & \\
\hline Approach & & & & $<.001^{\mathrm{c}}$ \\
\hline Open & $41(23.8)$ & $27(8.7)$ & $68(14.0)$ & \\
\hline Scopic & $131(76.2)$ & $285(91.3)$ & $416(86.0)$ & \\
\hline Stoma & & & & $.370^{\mathrm{c}}$ \\
\hline No stoma & $114(66.3)$ & $230(73.7)$ & $344(71.1)$ & \\
\hline Loop ileostomy & $31(18.1)$ & $51(16.4)$ & $82(16.9)$ & \\
\hline End colostomy & $27(16.7)$ & $31(10.0)$ & $58(12.0)$ & \\
\hline Urgency & & & & $.231^{\mathrm{c}}$ \\
\hline Elective & $150(87.2)$ & $283(90.7)$ & $433(89.5)$ & \\
\hline Emergency & $22(14.7)$ & $29(9.3)$ & $51(10.5)$ & \\
\hline Dukes classification & & & & $.735^{\mathrm{c}}$ \\
\hline Dukes' A & $0(0)$ & $3(1.0)$ & $127(26.2)$ & \\
\hline Dukes' B & $42(24.4)$ & $82(26.3)$ & $166(34.3)$ & \\
\hline Dukes' C & $54(31.4)$ & $102(32.7)$ & $142(29.3)$ & \\
\hline Dukes' D & $3(1.7)$ & $7(2.2)$ & $45(9.3)$ & \\
\hline Resection status & & & & $.002^{\mathrm{c}}$ \\
\hline $\mathrm{R} 0$ & $155(90.1)$ & $308(98.7)$ & $463(96.9)$ & \\
\hline $\mathrm{R} 1$ & $10(5.8)$ & $2(0.6)$ & $12(2.5)$ & \\
\hline $\mathrm{R} 2$ & $1(0.6)$ & $2(0.6)$ & $3(0.6)$ & \\
\hline Total & $172(100)$ & $312(100)$ & $484(100)$ & \\
\hline
\end{tabular}

${ }^{\text {a }}$ Unless otherwise stated in the first column

${ }^{\mathrm{b}}$ Mann-Whitney $U$ test

${ }^{\mathrm{c}}$ Chi-square test

before 2011 to 6.8 days after 2011 ( $p=.553$; median 7 vs. 7 days) (Table 3). The number of CTs performed before and after the implementation of the QIP was comparable; before implementation of the QIP, a CT scan was performed in 29 out of 172 patients $(19.8 \%)$. Of these CTs, 11 were negative $(38.0 \%)$. After implementation of the QIP, 54 CTs were performed, which was $17.3 \%$ of the total operated population $(n=312)$. Of these CTs, 32 were negative $(59.3 \%)$. 
Table 3 Outcomes

\begin{tabular}{|c|c|c|c|c|}
\hline & $\begin{array}{l}\text { Pre-implementation } \\
N(\%)^{\mathrm{a}}\end{array}$ & $\begin{array}{l}\text { Post-implementation } \\
N(\%)^{\mathrm{a}}\end{array}$ & $\begin{array}{l}\text { Total } \\
N(\%)^{\mathrm{a}}\end{array}$ & $p$ value \\
\hline Mortality & $15(8.7)$ & $8(2.6)$ & $23(4.7)$ & $.003^{\mathrm{c}}$ \\
\hline Complication Clavien-Dindo $>3 b$ & $43(25.0)$ & $43(13.7)$ & $86(17.1)$ & $.001^{\mathrm{c}}$ \\
\hline Anastomotic Leak & $17(9.8)$ & $13(4.2)$ & $30(6.4)$ & $.013^{\mathrm{c}}$ \\
\hline Complication Clavien-Dindo $<3 b$ & $42(24.4)$ & $79(25.3)$ & $117(24.1)$ & $.474^{\mathrm{c}}$ \\
\hline Number of admission days (SE) & $15.7(19.7)$ & $10.0(9.3)$ & $12.0(14.1)$ & $<.001^{\mathrm{b}}$ \\
\hline Number days operation - CT (SE) & $9.0(6.8)$ & $6.0(2.2)$ & $6.7(5.2)$ & $.553^{\mathrm{b}}$ \\
\hline Number of days operation - re-intervention (SE) & $9.6(6.8)$ & $6.8(2.3)$ & $7.4(4.5)$ & $.546^{\mathrm{b}}$ \\
\hline
\end{tabular}

${ }^{a}$ Unless otherwise stated in the first column

${ }^{\mathrm{b}}$ Mann-Whitney $U$ test

${ }^{\mathrm{c}}$ Chi-square test

Failure-to-rescue rates, defined as mortality among patients with serious complications, decreased from $34.9 \%$ before implementation of the program to $15.0 \%$ after implementation $(p=.039)$.

\section{Discussion}

This study highlights the effect of implementing a quality improvement program in a non-academic, non-referral community hospital on surgical outcomes. A significant decrease in mortality, anastomotic leakage, and other major complications was found, with a significant decrease in length of hospital stay after implementation of the QIP program.

The interventions introduced were intended to improve our results with regard to anastomotic leakage, mortality, and other major complications. A multivariate analysis showed that patients operated before implementation of the program had a significant higher risk for a major complication with an odds ratio of 0.537 . No difference was observed between the two cohorts regarding minor complication. This corroborates the conclusion that the program made a significant difference in preventing major complications.

Unfortunately, it was not possible to investigate the errors in the first two interventions after implementation of the program. Accidently, NSAID usage was not well documented, and the verifying of fluid management charts was not feasible; meaning that although it was protocolized, we could not check whether the protocol was indeed applied. Therefore, it is difficult to point out which separate intervention could be responsible for these improved results. It is unlikely that prevention of major complications in colorectal surgery is dependent on 1 or 2 factors. Rather, it is our belief that the improved results can be ascribed to a wide variety of measures [25].

The use of NSAIDS for postoperative pain management after colorectal surgery is still under debate. Currently, there is circumstantial evidence that there is a link to anastomotic leakage [26-28]. NSAIDS pre- and postoperatively as
Table 4. Multivariate analysis; complications Clavien-Dindo $>3 b$

\begin{tabular}{lllllll}
\hline & $\begin{array}{l}\text { Univariate } \\
\text { Odds ratio }\end{array}$ & $\begin{array}{l}\text { Univariate } \\
95 \% \mathrm{CI}\end{array}$ & $p$ value & $\begin{array}{l}\text { Multivariate } \\
\text { Odds ratio }\end{array}$ & $\begin{array}{l}\text { Multivariate } \\
95 \% \mathrm{CI}\end{array}$ & $p$ value \\
\hline Age & 1.03 & $1.01-1.05$ & .003 & & $\mathrm{~ns}$ \\
Sex & 0.86 & $0.53-1.39$ & $\mathrm{~ns}$ & & & $\mathrm{ni}$ \\
ASA 1-2 vs. 3-4 & 2.72 & $1.63-4.55$ & .001 & 2.57 & $1.50-4.38$ & .001 \\
Type of operation & 1.19 & $1.00-1.43$ & .053 & & & $\mathrm{~ns}$ \\
Open vs. scopic & 0.30 & $0.17-0.54$ & $<.001$ & 0.50 & $0.27-0.92$ & .027 \\
Emergency vs. elective & 2.50 & $1.31-4.77$ & .006 & & & $\mathrm{~ns}$ \\
Before vs. after 2011 & 0.44 & $0.27-0.71$ & .001 & 0.53 & $0.32-0.88$ & .014 \\
Stoma vs. no stoma & 2.56 & $1.58-4.17$ & $<.001$ & 2.14 & $1.27-3.61$ & .004 \\
R0 vs R1-2 & 0.353 & $0.05-2.72$ & $\mathrm{~ns}$ & & & $\mathrm{ni}$ \\
Dukes'A-B vs. C-D & 1.03 & $0.63-1.68$ & $\mathrm{~ns}$ & & & $\mathrm{ni}$ \\
\hline
\end{tabular}

$n s$ non significant, $n i$ not included 
postoperative pain management did not seem an absolute necessity and instead oral opioids were used.

To this day, intra-operative fluid management remains an area of debate. The concept that fluid restriction or avoidance of fluid overload in major abdominal surgery influences perioperative outcomes, such as morbidity and length of stay, is getting more support. However, it remains unclear whether this is related to the fluid restriction, per se, or the maintenance of a state of zero fluid balance [29]. In the QIP program, a 24-h zero fluid balance was pursued versus no restriction before implementation of the program.

Another important intervention was fast re-intervention by a surveillance protocol with an abdominal CT-scan with rectal contrast was performed when infection parameters (CRP) and/ or clinical symptoms where suspect of a complication. Presently, a re-intervention within 4 days after surgery is strived for in our institution. While this does not influence the major complication rate, it does decrease the subsequent sequel, resulting in shorter hospital stay and lower the failureto-rescue rates and, by that, mortality. Strikingly, this change in protocol did not result in an increased percentage of CT's (19.8 vs. $17.3 \%)$.

The number of elderly patients presenting with obstructive colorectal pathology is still increasing. These patients are at high risk for complications or death. A two-staged procedure, first deflating the colon with a colostomy and then revitalizing the patient before definitive surgery, has been beneficial to the improvement in results in patient's ages 80 years and over.

This study is limited by the retrospective character of the study and the fact that it concerns a single-center study. However, for analyzing the effect of the introduction of a quality improvement program, a homogenous patient group from a single center is more suitable, with a relatively large series and little missing data. Almost all remaining factors were consistent during the timeframe of this study. Obviously, the effect of a learning curve can not be excluded, but over the years, there was no change in the surgical team consisting of all dedicated colorectal surgeons with over 3 years of experience before the introduction of the quality program. In addition, the patient inflow remained consistent, and there were no other changes in postoperative patient management.

It is also acknowledged that in any non-randomized study, even when experimental and control groups appear comparable at baseline, the effect size estimate is still at risk of bias due to residual confounding. One of the largest confounding factors for this study is the concept that results might improve over time due to the surgical learning curve of each surgeon. However, as mentioned, surgeons remained consistent and even when laparoscopic versus open procedure was included in the multivariate analysis, the decrease in major complications after introducing the program remained significant.

\section{Conclusion}

A significant decrease in major complication rates and mortality was observed after careful re-evaluation of national auditing results and introducing this quality improvement program.

This study showed that these relatively easy interventions could be safely implemented and be promising for other hospitals.

\section{Compliance with ethical standards}

Funding None.

Financial disclosures None of the authors of this study has financial or other relationships that may cause a conflict of interest.

Open Access This article is distributed under the terms of the Creative Commons Attribution 4.0 International License (http:// creativecommons.org/licenses/by/4.0/), which permits unrestricted use, distribution, and reproduction in any medium, provided you give appropriate credit to the original author(s) and the source, provide a link to the Creative Commons license, and indicate if changes were made.

\section{References}

1. Longo WE, Vorgo KS, Johnson FE, et al. (2000) Risk factors for morbidity and mortality after colectomy for colon cancer. Dis Colon Rectum 43:83-91

2. Alves A, Panis Y, Mathieu P, et al. (2005) Postoperative mortality and morbidity in French patients undergoing colorectal surgery: results of a prospective multicenter study. Arch Surg 140(3):278283

3. Ragg JL, Watters DA, Guest GD (2009) Preoperative risk stratification for mortality and major morbidity in major colorectal surgery. Dis Colon Rectum 52(7):1296-1303. doi:10.1007/DCR.0 b013e3181a0e639

4. Van Leersum NJ, Snijders HS, Henneman D, et al. (2003) The Dutch surgical colorectal audit. Eur J Surg Oncol 39(10):10631070. doi:10.1016/j.ejso.2013.05.008

5. Koca A, Sen C (2006) Effects of diclofenac sodium on bursting pressures of anastomoses and hydroxyproline contents of perianastomotic tissues in a laboratory study. Int J Surg 4(4):222227

6. Klein M, Andersen LPH, Harvald T, et al. (2009) Increased risk of anastomotic leakage with diclofenac treatment after laparoscopic colorectal surgery. Dig Surg 26(1):27-30. doi:10.1159/000193329

7. Holte K, Andersen J, Jakobsen DH, et al. (2009) Cyclo-oxygenase 2 inhibitors and the risk of anastomotic leakage after fast-track colonic surgery. Br J Surg. 96(6):650-654

8. Lobo DN, Bostock KA, Neal KR, et al. (2002) Effect of salt and water balance on recovery of gastrointestinal function after elective colonic resection: a randomised controlled trial. Lancet 359(9320): 1812-1818

9. Noblett SE, Snowden CP, Shenton BK, et al. (2006) Randomized clinical trial assessing the effect of Doppler-optimized fluid management on outcome after elective colorectal resection. Br J Surg 93(9):1069-1076

10. Rahbari NN, Zimmermann JB, Schmidt T, et al. (2009) Metaanalysis of standard, restrictive and supplemental fluid 
administration in colorectal surgery. Br J Surg 96(4):331-341. doi:10.1002/bjs.6552

11. Tan KY, Kawamura Y, Mizokami K, et al. (2009) Colorectal surgery in octogenarian patients-outcomes and predictors of morbidity. Int J Color Dis 24(2):185-189. doi:10.1007/s00384-008-0615-9

12. Smothers L, Hynan L, Fleming J, et al. (2003) Emergency surgery for colon carcinoma. Dis Colon Rectum 46(1):24-30

13. Kronborg O (1995) Acute obstruction from tumour in the left colon without spread. A randomised trial of emergency colostomy versus resection. Int J Color Dis 10(1):1-5

14. Trompetas V (2008) Emergency management of malignant acute left-sided colonic obstruction. Ann R Coll Surg Engl 90(3):181186. doi: $10.1308 / 003588408 X 285757$

15. Woeste G, Muller C, Bechstein WO, et al. (2010) Increased serum levels of C-reactive protein precede anastomotic leakage in colorectal surgery. World J Surg 34(1):140-146. doi:10.1007/s00268-0090304-Z

16. den Dulk M, Noter SL, Hendriks ER, et al. (2009) Improved diagnosis and treatment of anastomotic leakage after colorectal surgery. Eur J Surg Oncol 35(4):420-426. doi:10.1016/j.ejso.2008.04.009

17. Kehlet H, Wilmore DW (2008) Evidence-based surgical care and the evolution of fast-track surgery. Ann Surg 248(2):189-198. doi:10.1097/SLA.0b013e31817f2c1a

18. Eskicioglu C, Forbes SS, Fenech DS, et al. (2010) Best practice in General Surgery Committee. Preoperative bowel preparation for patients undergoing elective colorectal surgery: a clinical practice guideline endorsed by the Canadian Society of Colon and Rectal Surgeons. Can J Surg 53(6):385-395

19. Guenaga KK, Matos D, Wille-Jørgensen P. (2009) Mechanical bowel preparation for elective colorectal surgery. Cochrane Database Syst Rev. (1):CD001544. doi: 10.1002/14651858.CD001544.pub3.

20. Biondo S, Kreisler E, Millan M, et al. (2010) Impact of surgical specialization on emergency colorectal surgery outcomes. Arch Surg 145(1):79-86. doi:10.1001/archsurg.2009.208
21. Miles WE (1971) A method of performing abdomino-perineal excision for carcinoma of the rectum and of the terminal portion of the pelvic colon (1908). CA Cancer J Clin 21(6):361-364

22. Bergqvist $\mathrm{D}$ (2006) Venous thromboembolism: a review of risk and prevention in colorectal surgery patients. Dis Colon Rectum 49(10): $1620-1628$

23. Dindo D, Demartines N, Clavien PA (2004) Classification of surgical complications: a new proposal with evaluation in a cohort of 6336 patients and results of a survey. Ann Surg 240(2):205-213

24. Silber JH, Rosenbaum PR, Schwartz JS, et al. (1995) Evaluation of the complication rate as a measure of quality of care in coronary artery bypass graft surgery. JAMA 274(4):317-323

25. McDermott FD, Heeney A, Kelly ME, et al. (2015) Systematic review of preoperative, intraoperative and postoperative risk factors for colorectal anastomotic leaks. Br J Surg 102(5):462-479. doi:10.1002/bjs.9697

26. Bhangu A, Singh P, Fitzgerald JE, et al. (2014) Postoperative nonsteroidal anti-inflammatory drugs and risk of anastomotic leak: meta-analysis of clinical and experimental studies. World J Surg 38(9):2247-2257. doi:10.1007/s00268-014-2531-1

27. Saleh F, Jackson TD, Ambrosini L, et al. (2014) Perioperative nonselective non-steroidal anti-inflammatory drugs are not associated withanastomotic leakage after colorectal surgery. J Gastrointest Surg 18(8):1398-1404. doi:10.1007/s11605-014-2486-4

28. Hakkarainen TW, Steele SR, Bastaworous A, et al. (2015) Nonsteroidal anti-inflammatory drugs and the risk for anastomotic failure: a report from Washington State's Surgical Care and Outcomes Assessment Program (SCOAP). JAMA Surg 150(3): 223-228. doi:10.1001/jamasurg.2014.2239

29. Eng OS, Melstrom LG, Carpizo DL (2015) The relationship of perioperative fluid administration to outcomes in colorectal and pancreatic surgery: a review of the literature. J Surg Oncol 111(4): 472-477. doi:10.1002/jso.23857 